\title{
Acute kidney injury in critically ill
}

\author{
K Raveendran ${ }^{1^{*}}$ \\ Consultant in Anaesthesia and Intensive Care \\ Barking Havering and Redbridge University Hospitals, Essex, United Kingdom.
}

\begin{abstract}
Acute kidney injury (AKI) is frequent in hospitalised critically ill patients with a high incidence in ICU patients. It is associated with increased mortality, greater cost, prolonged intensive care and hospital stay and progress to development of chronic kidney disease. AKI is an independent risk factor for increased mortality and severe morbidity. Sepsis is a leading cause of AKI in critically ill patients. The management is still supportive and early recognition, haemodynamic optimisation, avoidance of nephrotoxic medications, treatment of the underlying cause and renal replacement therapy are important.

AKI is a preventable and outcome can be improved by early diagnosis and effective management.

The aim of this review is to provide a comprehensive update on recent evidence in the field of AKI in critically ill patients, including risk factors, causes, pathophysiology, diagnosis/classification, prevention and management.
\end{abstract}

Keywords: Acute kidney injury; critically ill; renal biomarkers; renal replacement therapy

\section{Introduction}

Acute kidney injury (AKI) is frequent in hospitalized critically ill patients and occurs in approximately $36 \%$ of intensive care patients. Mortality associated with AKI has remained unchanged over many decades and about one third of patients progress to develop chronic kidney disease. It is associated with increased mortality, greater cost, and prolonged intensive care and hospital stay. AKI is an independent risk factor for increased mortality and severe morbidity. ${ }^{1}$

The cause of AKI in critically ill patients is often multi factorial and sepsis is the lead contributing factor. At present, the management of AKI remains supportive and key principles of management include early recognition,

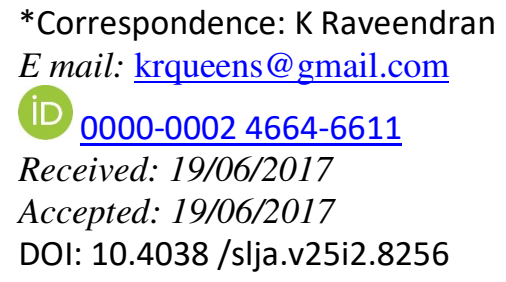

*Correspondence: K Raveendran

E mail: krqueens@gmail.com

0000-0002 4664-6611

Received: $19 / 06 / 2017$

Accepted: 19/06/2017

DOI: 10.4038 /slja.v25i2.8256

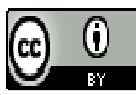

haemodynamic optimisation, ceasing and avoidance of nephrotoxic medications, treatment of the underlying cause and renal replacement therapy.

AKI is a preventable clinical entity and incidence can be reduced by identifying patients who are at risk and taking adequate measures to minimise and prevent AKI. Outcome can be improved by early diagnosis and effective management.

\section{Classification}

The definition of AKI has evolved from the Risk, Injury, Failure, Loss, End-stage (RIFLE) criteria in 2004 to the Acute Kidney Injury Network (AKIN) classification in 2007.,3 In 2012, both were merged resulting in the Kidney Disease Improving Global Outcomes (KDIGO) classification. ${ }^{4}$

AKI based on the presence of at least one of 3 following diagnostic criteria (KDIGO)

Increase in SCr by $0.3 \mathrm{mg} / \mathrm{dl}$ (26.5 micro mol/l) within 48 hours; or

Increase in $\mathrm{SCr}$ to 1.5 times baseline, which is known or presumed to have occurred within the prior 7 days; or

Urine volume $0.5 \mathrm{ml} / \mathrm{kg} / \mathrm{h}$ for 6 hours. 


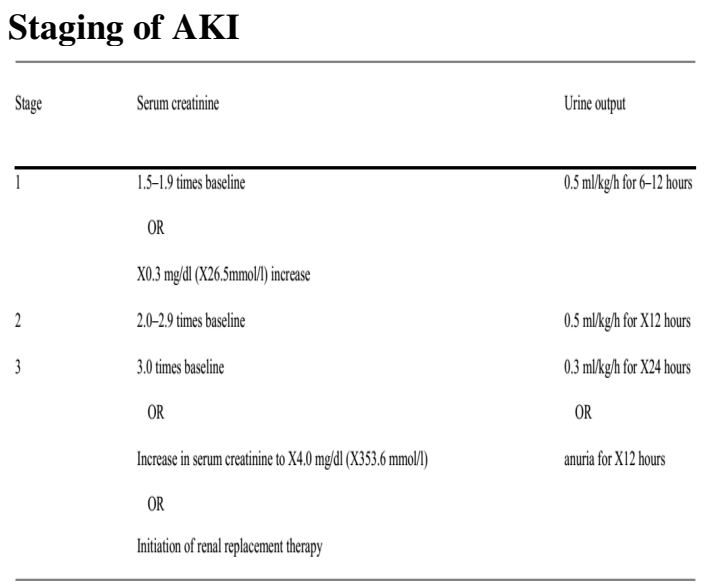

Risk factors and recognition

Early identification and prevention of AKI is vital as it is associated with a high mortality and the treatment is only supportive. Therefore, it is important to look for risk factors for AKI in hospitalized patients in order to intervene early. There are significant numbers of risk factors for AKI, which are patient or procedure related.

Kheterpal et $\mathrm{al}^{5}$ designed (“AKI Risk Index”) to predict the development of AKI after noncardiac major surgery. This score is based on the allocation of points for each risk factors as follows: age $\geq 56$ years, male sex, cardiac failure, ascites, hypertension, emergency surgery, abdominal surgery, chronic renal insufficiency (CRI) and diabetes. The greater the number of risk factors, the greater the risk of AKI. The two most important risk factors are age and preexisting chronic kidney disease. Among procedure related risk factors, the most frequently responsible are sepsis and surgery. The incidence of AKI increased from $0.2 \%$ in patients with 0-2 risk factors to $9.5 \%$ in those with more than five risk factors, and this incidence increased with the number of risk factors.

\section{Pathophysiology}

AKI is now considered to be a broad clinical syndrome encompassing various aetiologies, including pre-renal, renal, and post renal. The commonest pre-renal cause is hypoperfusion leading to acute tubular necrosis. Renal causes include sepsis, acute nephritis and vasculitic diseases. Sepsis has emerged as the most important and prevalent predictor in critically ill patients. The commonest post renal cause is obstructive nephropathy.

In pre-renal AKI, the renin-angiotensinaldosterone system, the renal sympathetic system, and the tubulo -glomerular feed back system are activated due to hypoperfuson. These circulatory changes induce renal vasoconstriction and lead to retention of salt and water. Impaired renal blood flow with little relevance to period of hypoperfusion can lead to hypoxic injury to the renal tubular cells by infiltration of leukocytes, injuring the endothelium, releasing cytokines and causing apoptosis. $^{6}$

Until recently, sepsis-induced AKI was considered to be a result of hypoperfusion induced renal ischaemia, cellular damage and acute tubular necrosis (ATN). However, recent evidence suggests that AKI in sepsis can occur in the absence of hypoperfusion ${ }^{7}$ and the glomerular filtration rate (GFR) decreases rapidly despite normal or increased cardiac output and a hyperdynamic circulation. These data support that mechanisms other than tissue hypoperfusion are involved and the mechanisms that are believed to be responsible are microcirculatory dysfunction, tubular cell damage caused by cytokines, innate and acquired immune-mediated injury, and cell apoptosis. In addition, septic AKI is associated with higher mortality and longer ICU and hospital stay when compared with non-septic AKI.

In obstructive nephropathy, back pressure on the kidney can result in decreased renal blood flow and GFR. This in turn can cause atrophy and apoptosis of renal tubules and interstitial fibrosis. If left untreated obstructive nephropathy can cause irreversible renal damage.

\section{Diagnosis of AKI}

The clinical evaluation of AKI includes a careful history focusing on risk factors and thorough physical examination. The decrease in urine output and the increase in traditional renal biomarkers, such as blood urea nitrogen (BUN) and serum creatinine (Scr), have largely been used as surrogate markers for decreased glomerular filtration rate (GFR), which defines AKI. Despite having several limitations, SCr remains the dominant renal biomarker used in 
the current definitions of AKI since it is easy to measure and being standardised for a long time.

$\mathrm{SCr}$ and plasma urea concentrations are insensitive markers of glomerular filtration rate, as they are modified by nutrition, gastrointestinal bleeding, corticosteroid therapy, high protein diet, muscle mass, age, sex and aggressive fluid resuscitation. $^{8}$ Indeed, increased levels of creatinine are observed only when the glomerular filtration rate decreases by more than $50 \%$.

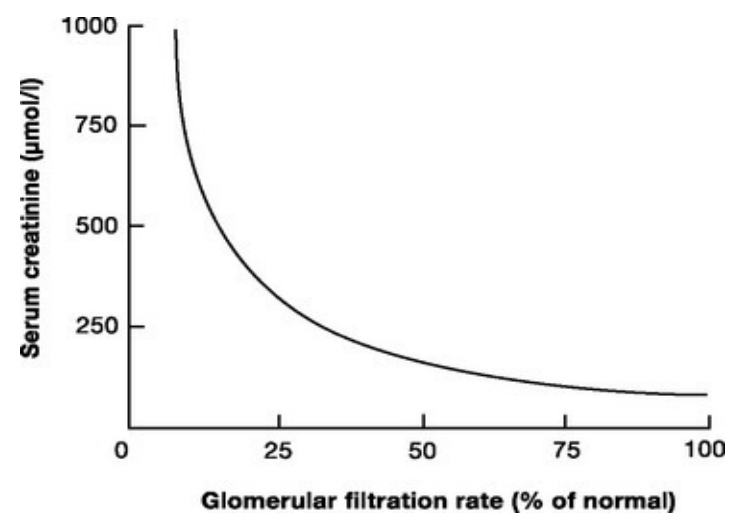

Relationship between serum creatinine and GFR

Serum electrolytes, urine analysis, and imaging studies should be performed as a minimum, with additional tests depending on the clinical presentation.

Renal ultrasonography is useful for evaluating existing structural renal disease and diagnosing obstruction of the urinary collecting system. In particular, the presence of reduced corticomedullary differentiation and decreased kidney size is indicative of underlying CKD.

\section{Use of new biomarkers in the diagnosis of} AKI

There have been numerous studies in the literature evaluating new biomarkers for early diagnosis of AKI. Among these biomarkers, neutrophil gelatinase-associated lipocalin (NGAL) and Cystatin C are the most frequently studied. These markers seem to change earlier than $\mathrm{sCr}$ concentrations, by showing different aspects of renal injury. For example, Cystatin C concentrations are related to changes in glomerular filtration rate, ${ }^{9}$ whereas concentrations of NGAL are related to tubular stress or injury. ${ }^{10}$ Since these various biomarkers indicate different mechanisms of injury and their syntheses are located in different sites, and they are activated with different kinetics following kidney injury ${ }^{10}$ despite growing literature, there is no study that truly demonstrates their utility in clinical practice for critically ill patients at risk of AKI.

\section{Causes of AKI in critically ill}

\section{Nephrotoxic agents}

Major nephrotoxic agents responsible for AKI are radiocontrast agents, aminoglycosides, amphotericin, non-steroidal anti-inflammatory agents, $\beta$-lactams (interstitial nephropathies) sulfamides, aciclovir, methotrexate, cyclosporine, tacrolimus, angiotensinconverting-enzyme inhibitors (ACE).

If clinical situation warrants, nephrotoxic agents should be replaced by non-nephrotoxic agents or administered using associated preventive measures. For example, adhering to single dosing aminoglycosides with close monitoring of levels and restricting them for maximum of 3 days whenever possible has been recommended. ${ }^{11}$

Avoiding non-steroidal anti-inflammatory drugs (NSAIDs), angiotensin converting enzyme inhibitors (ACEIs), and angiotensin 2 receptor antagonists in patients at risk of AKI also has been recommended. ${ }^{11}$

\section{Contrast Induced Nephropathy and prevention (CIN)}

Acute deterioration of renal function after intravenous administration of radio contrast media is referred to as contrast-induced nephropathy (CIN) and is generally defined as an increase in serum creatinine concentration of more than $0.5 \mathrm{mg} / \mathrm{dl}$ (44 micromole/L) or $25 \%$ above baseline within 48 hours after contrast administration. $^{12}$

It has been shown that optimisation of hydration and volume status with crystalloids before giving contrast media and continued hydration for 6 $12 \mathrm{hrs}$ after, is the most efficient and effective measure in preventing CIN. The safety and efficacy of intravenous $\mathrm{N}$ Acetyl Cysteine (NAC) in this specific population has not been 
established. The risk of developing CIN is largely determined by base line renal function and by the volume and osmolarity of radio contrast agent given. ${ }^{12}$ Therefore the use of lowosmolar or iso-osmolar contrast medium and the lowest volume as possible has been recommended.

\section{Tumour Lysis Syndrome}

Tumour lysis syndrome refers to the metabolic derangements that result from the rapid destruction of malignant cells and the abrupt release of intracellular ions, nucleic acids, proteins and their metabolites into the extracellular space after the initiation of cytotoxic therapy. Risk factors for tumour lysis syndrome include lymphoproliferative malignancies highly sensitive to chemotherapy, pre-existent renal dysfunction, and treatment with nephrotoxic agents. Metabolic disorders that occur in tumour lysis syndrome include hypocalcaemia, hyperuricaemia, hyperkalaemia, metabolic acidosis, and AKI and hyperphosphataemia. ${ }^{13}$

In established tumour lysis syndrome, management of electrolyte abnormalities, aggressive hydration and renal replacement therapy (RRT) to remove uric acid, phosphate and potassium, and correction of azotaemia, are the main supportive measures.

\section{Rhabdomyolysis}

Rhabdomyolysis (RM) is a clinical syndrome characterized by injury to skeletal muscle fibers with disruption and release of their contents into the circulation which can be followed by acute kidney injury (AKI). Myoglobin, creatine phosphokinase (CK) and lactate dehydrogenase are the most important substances for indicating muscle damage and myoglobin has been shown to be the main agent responsible of AKI.

The three different mechanisms of renal toxicity by myoglobin are renal vasoconstriction, formation of intra tubular casts and the direct toxicity of myoglobin to kidney tubular cells. ${ }^{14}$ Renal vasoconstriction is caused by reduced renal blood flow due to excessive leakage of extracellular fluid into the damaged muscle cells and by secondary activation of the reninangiotensin-aldosterone system. However, a second theory favours the effect of the nitric oxide scavenging characteristics of myoglobin and release of cytokines. ${ }^{14}$

The commonly used conservative measures include massive hydration, use of mannitol, urine alkalization and forced diuresis. Early and aggressive fluid resuscitation to restore renal perfusion and increase the urine flow rate is agreed on as the main intervention for preventing and treating AKI. ${ }^{15}$ Use of bicarbonate has not been shown to be superior to saline diuresis in increasing urine $\mathrm{pH}>6.5-7$. However, if the patient is severely oliguric or anuric, renal replacement therapy needs to be initiated without delay.

\section{Abdominal compartment syndrome \\ Recent evidence has implicated intra abdominal hypertension (IAH) as an independent risk factor for acute kidney injury (AKI). In intensive care populations, the incidence of IAH and abdominal compartment syndrome may be as high as $54 \%$ and $12 \%$, respectively. ${ }^{16}$}

Factors predisposing to IAH/ACS include sepsis, large volume fluid resuscitation, ileus, abdominal surgery, mechanical ventilation with high intrathoracic pressure and ascites, among others.

Normal intra-abdominal pressure generally is $\leq 5 \mathrm{mmHg}$. IAH is defined as sustained intraabdominal pressure $\geq 12 \mathrm{mmHg}$, and abdominal compartment syndrome is defined as abdominal pressures $>18 \mathrm{mmHg}$. IAH and abdominal compartment syndrome can lead to multi organ dysfunction. ${ }^{16}$

The concept of abdominal perfusion can be defined as the difference between mean arterial pressure and intra-abdominal pressure. As IAH increases, abdominal perfusion pressure decreases, and this in turn causes a decrease in kidney perfusion pressure. The maintenance of an APP of $\geq 50 \mathrm{mmHg}$ seems to provide proper intra-abdominal circulation. ${ }^{17}$

Apart from the decreased kidney perfusion induced by low arterial perfusion, elevated intra abdominal pressure also can cause renal vein compression, which impairs venous drainage, and leads to a progressive decrease in glomerular perfusion. This decrease in glomerular filtration 
gradient appears to be the key causative factor in IAH-induced AKI.

Common practice for the screening of patients at risk of IAH is the serial monitoring of IAP every 4-6 hrs. If the trend is on the increase, interventions to reduce IAH should be implemented without delay.

Rapid reversal of AKI can be achieved by reducing/relieving intra abdominal hypertension. There are some simple nonsurgical measures that can possibly reduce IAP to varying extents. These are aimed at improving abdominal wall compliance and include gastrointestinal tract evacuation and decompression, draining any intra-abdominal fluid, abscess, or blood, and minimizing the capillary leak process with antibiotic therapy for sepsis.

In fully established ACS, decompressive laparotomy remains the treatment of choice. Early, rather than late, decompression is gaining more popularity and is associated with better outcomes.

\section{Hepato renal syndrome (HRS)}

HRS is the development of AKI in patients with advanced chronic liver disease who have portal hypertension and ascites. Estimates indicate that at least $40 \%$ of patients with cirrhosis and ascites will develop HRS during the natural history of their disease.

In HRS, the histological appearance of the kidneys is normal, and the kidneys often resume normal function following liver transplantation. The diagnosis of the hepato renal syndrome is one of exclusion, and other potential causes of acute or sub acute kidney injury need to be ruled out first.

The pathogenesis of HRS is not fully understood. An interplay between disturbances in systemic haemodynamics, activation of vasoconstrictor systems, and a reduction in the activity of the vasodilator systems are some of the mechanisms believed to be involved. The haemodynamic pattern of patients with HRS is characterized by increased cardiac output, low arterial pressure, and reduced systemic vascular resistance. Renal vasoconstriction occurs in the absence of reduced cardiac output and blood volume, which is in contrast to most clinical conditions associated with renal hypoperfusion. ${ }^{18}$ There are 2 different forms of HRS described in the literature. ${ }^{17}$ Although their pathophysiology is similar, their manifestations and outcomes are different.

Type 1 HRS is characterized by rapid and progressive renal impairment and the most common cause is spontaneous bacterial peritonitis (SBP). Type 1 HRS occurs in approximately $25 \%$ of patients with SBP, and is associated with high mortality. Type 2 HRS is characterized by a moderate and stable reduction in the GFR and commonly occurs in patients with relatively preserved hepatic function. These patients have a better outcome than type 1 HRS with a reduced mortality.

Early recognition and treatment of sepsis, hypotension, elevated abdominal compartment pressures, and avoidance of nephrotoxins such as aminoglycosides, when possible, are of primary importance in order to prevent and minimise HRS.

Albumin volume expansion reduces renal risks in patients with peritonitis and during therapeutic paracentesis for tense ascites. Prompt intervention for HRS including vasopressors and albumin may reverse renal dysfunction.

The ideal therapy for hepatorenal syndrome is improvement of liver function from recovery of alcoholic hepatitis, treatment of decompensated hepatitis B with effective antiviral therapy, recovery from acute hepatic failure, or liver transplantation.

\section{AKI management}

The clinical consequences of AKI include accumulation of waste products, electrolytes, and fluid, but also less obvious effects, including reduced immunity and dysfunction of non-renal organs: cardiac, respiratory, and cerebral (organ cross-talk). ${ }^{19}$

Early stages of renal injury may be still reversible. Urgent measures should be taken to reverse factors that have caused or contributed to renal dysfunction such as discontinuation of nephrotoxic drugs, treatment of sepsis and 
restoration of renal blood flow and homeostasis. It is of key importance to avoid hypovolaemia, and fluid resuscitate adequately aiming to maintain a mean arterial pressure (MAP) of 65$70 \mathrm{mmHg}$ with vasopressors if required. Crystalloids are preferable over colloids as some colloids can worsen the renal damage. Several studies have shown potentially deleterious effects of non-balanced solutions associated with hyperchloraemia especially for kidneys, and suggest using balanced solutions especially for large-volume resuscitation. ${ }^{20}$ Diuretics to prevent or treat AKI is not recommended and should only be used to treat fluid overload. There is no evidence in the literature to support the use of mannitol, dopamine, NAC, in the prevention or treatment of AKI.

In the absence of any effective pharmacologic therapies for AKI, its management remains supportive, focused on optimising fluid balance, maintaining nutrition, preventing or treating electrolyte and acid-base disturbances, adjusting the dosing of medications that are excreted by the kidney, and avoiding secondary haemodynamic and nephrotoxic renal injury.

\section{Timing of initiation of renal replacement therapy}

RRT aim at averting the immediately lifethreatening consequences of severe renal dysfunction, and allowing time for kidney recovery to occur. Conventional indications for RRT are volume overload, electrolyte and acidbase disturbances, severe hyperkalaemia, metabolic acidosis, and uraemic manifestations, such as pericarditis and encephalopathy. To date there is little evidence to guide the optimal timing of RRT for AKI. Recent meta analysis concluded that "early" initiation of RRT in critically ill patients with AKI does not improve survival. ${ }^{21}$ Although early initiation of RRT is not clearly associated with benefit, avoiding or delaying RRT is associated with higher mortality and increased hospital/ICU lengths of stay.

\section{When to stop renal replacement therapy}

There is an even greater paucity of data on when to stop RRT. RRT can be stopped when there is sufficient improvement in renal function, but how this can be evaluated while the patient is still receiving RRT remains unclear. Current practice suggests measuring urine output and serum creatinine levels while on a constant dose of CRRT and calculating the endogenous creatinine clearance by using both the urine and serum concentrations of creatinine. A urine output of more than $400 \mathrm{~mL} /$ day is a reasonable cut off value, resulting in correct classification in $79 \%$ of patients. ${ }^{22}$

\section{Prognosis}

AKI has a poor and variable prognosis and is influenced by underlying cause and co morbidities. Pre-AKI chronic kidney disease has been shown to be associated with doubling of mortality and four or five-fold increase in poor renal outcomes.

\section{Conclusion}

AKI is common in critically ill patients. Despite advances in the understanding of pathophysiology and management strategies, the mortality and morbidity associated with AKI remains high. Therefore, it is paramount to identify patients at risk and monitor them closely to detect AKI early to improve patient safety and outcome.

\section{References}

1. Lameire N, Van Biesen W, Vanholder R. Acute kidney injury. Lancet. 2008;372(9653):18631865. https://doi.org/10.1016/S0140-6736(08)61794-8

2. Bellomo R, Ronco C, Kellum JA, et al. Acute renal failure: definition, outcome measures, animal models, fluid therapy and information technology needs: the second international consensus conference of the acute dialysis quality initiative (ADQI) group. Crit Care. 2004;8: R20412. https://doi.org/10.1186/cc2872

PMid:15312219 PMCid:PMC522841

3. Mehta RL, Kellum JA, Shah SV, et al. Acute kidney injury network: report of an initiative to improve outcomes in acute kidney injury. Crit Care. 2007;11: R31.

https://doi.org/10.1186/cc5713 PMid:17331245 PMCid:PMC2206446

4. Kidney Disease: Improving Global Outcomes (KDIGO) Acute Kidney Injury Work Group. KDIGO clinical practice guideline for acute kidney injury. Kidney Int. 2012; 2: 1-138

5. Kheterpal S, Tremper KK, Heung M, et al. Development and validation of an acute kidney injury risk index for patients undergoing general sur gery: results from a national data set. Anesthesiology. 2009; 110:505-15 
https://doi.org/10.1097/ALN.0b013e3181979440 PMid:19212261

6. Bonventre JV, Yang L. Cellular pathophysiology of ischemic acute kidney injury. J Clin Invest. 2011 Nov;121(11):4210-21.

https://doi.org/10.1172/JCI45161

PMid:22045571 PMCid:PMC3204829Gomez H, Ince C, De Backer D, et al. A unified theory of sepsis-induced acute kidney injury: inflammation, microcirculatory dysfunction, bioenergetics, and the tubular cell adaptation to injury. Shock. 2014; 41:3-1122.

https://doi.org/10.1097/SHK.0000000000000052 PMid:24346647 PMCid:PMC3918942

7. Bellomo R, Kellum JA, Ronco C. Acute kidney injury. Lancet. 2012 Aug 25;380(9843):756-66 https://doi.org/10.1016/S0140-6736(11)61454-2

8. Ahlström A, Tallgren M, Peltonen S, Pettilä V. Evolution and predictive power of serum cystatin $\mathrm{C}$ in acute renal failure. ClinNephrol. 2004 Nov;62(5):344-50. https://doi.org/10.5414/CNP62344 PMid: 15571178

9. Devarajan P. Review: neutrophil gelatinaseassociated lipocalin: a troponin-like biomarker for human acute kidney injury. Nephrology (Carlton). 2010 Jun;15(4):419-28.

https://doi.org/10.1111/j.1440-1797.2010.01317.x PMid:20609093

10. Ichai C, Vinsonneau C, Souweine B et al. Acute kidney injury in the perioperative period and in intensive care units (excluding renal replacement therapies) Ann. Intensive Care 2016; 6(1):48 https://doi.org/10.1186/s13613-016-0145-5 PMid:27230984 PMCid:PMC4882312

11. Barrett BJ, Parfrey PS. Clinical practice. Preventing nephropathy induced by contrast medium. N Engl J Med 2006; 354:379-386.

https://doi.org/10.1056/NEJMcp050801 PMid:16436769 Barrett BJ, Parfrey PS. Clinical practice. Preventing nephropathy induced by contrast medium. N Engl J Med 2006; 354:379386.

https://doi.org/10.1056/NEJMcp050801 PMid:16436769

12. Davidson MB, Thakkar S, Hix JK, Bhandarkar ND, Wong A, Schreiber MJ. Pathophysiology, clinical consequences, and treatment of tumor lysis syndrome. Am J Med 2004; 116:546-554 https://doi.org/10.1016/j.amjmed.2003.09.045 PMid: 15063817
13. Ronco C, Bellomo R, Kellum JA: Critical care nephrology. In Myoglobin as a toxin. 2nd edition. Philadelphia, PA: Saunders, Elsevier; 2009:11031109

14. Zimmerman JL, Shen MC: Rhabdomyolysis. Chest 2013, 144:1058-1065.

https://doi.org/10.1378/chest.12-2016

PMid:24008958

15. De Waele JJ, De Laet I, Kirkpatrick AW, Hoste E. Intraabdominal hypertension and abdominal compartment syndrome. Am $\mathrm{J}$ Kidney Dis. 2011;57(1):159-169.

https://doi.org/10.1053/j.ajkd.2010.08.034 PMid:21184922

16. Mohmand H, Goldfarb S. Renal dysfunction associated with intra-abdominal hypertension and the abdominal compartment syndrome. J Am Soc Nephrol. 2011; 22:615-621

https://doi.org/10.1681/ASN.2010121222

PMid:21310818

17. Turban S, Thuluvath PJ, Atta MG. Hepatorenal syndrome. World J Gastroenterol.2007 Aug 14 . 13(30):4046-55.

https://doi.org/10.3748/wig.v13.i30.4046

PMid:17696221 PMCid:PMC4205304

18. Singbartl K, Joannidis M. Short-term effects of acute kidney injury. Crit Care Clin. 2015;31(4):751-62.

https://doi.org/10.1016/j.ccc.2015.06.010

PMid:26410142

19. Raghunathan K, Murray PT, Beattie WS, ADQI XII Investigators Group, et al. Choice of fluid in acute illness: what should be given? An international consensus. $\mathrm{Br} \mathrm{J}$ Anaesth. 2014;113:772-83.

https://doi.org/10.1093/bja/aeu301

PMid:25326478

20. Wierstra BT, S Kadri, S Alomar et al. The impact of "early" versus "late" initiation of renal replacement therapy in critical care patients with acute kidney injury: a systematic review and evidence synthesis. Critical Care 2016; 20:122 https://doi.org/10.1186/s13054-016-1291-8 PMid:27149861 PMCid:PMC4858821

21. Uchino $\mathrm{S}$, Bellomo $\mathrm{R}$, Morimatsu $\mathrm{H}$, et al. Discontinuation of continuous renal replacement therapy: a post hoc analysis of a prospective multicenter observational study. Crit Care Med. 2009; 37:2576-2582)

https://doi.org/10.1097/CCM.0b013e3181a38241 PMid: 19623048 